\title{
CFD simulation of flow-induced vibration of an elastically supported airfoil
}

\author{
Petr Šidlof ${ }^{1, a}$ \\ ${ }^{1}$ Technical University of Liberec, NTI FM, Studentská 2, 46117 Liberec 1, Czech Republic
}

\begin{abstract}
Flow-induced vibration of lifting or control surfaces in aircraft may lead to catastrophic consequences. Under certain circumstances, the interaction between the airflow and the elastic structure may lead to instability with energy transferred from the airflow to the structure and with exponentially increasing amplitudes of the structure. In the current work, a CFD simulation of an elastically supported NACA0015 airfoil with two degrees of freedom (pitch and plunge) coupled with 2D incompressible airflow is presented. The geometry of the airfoil, mass, moment of inertia, location of the centroid, linear and torsional stiffness was matched to properties of a physical airfoil model used for wind-tunnel measurements. The simulations were run within the OpenFOAM computational package. The results of the CFD simulations were compared with the experimental data.
\end{abstract}

\section{Introduction}

In aerospace engineering, fluid-structure interaction can play a very important and potentially dangerous role: under certain circumstances, the coupling between flow and structure may lead to unstable exponentially increasing oscillations. The classical example is the flutter instability of airfoils, which occurs for systems with two degrees of freedom when the critical flow velocity is surpassed [1]. Flutter is a dynamic instability of an elastic structure coupled to airflow, caused by the interaction between elastic, inertial and aerodynamic forces. Flutter may occur at certain flow velocities and structural natural vibration frequencies, when the energy is transferred from the airflow to the structure and the internal damping is not able to absorb it. This can lead to vibration of an aircraft component with exponentially increasing amplitudes and catastrophic consequences. Thus, the aircraft components (wings, flaps and ailerions, stabilators, elevons and rudders) must be designed and tested not only to sustain dynamic loads induced by mechanical accelerations and air turbulence during takeoff, cruise and landing, but also to ensure that within design flight conditions, the aircraft components may never encounter dynamic flow-induced instability.

In general, flutter instability is a complex nonlinear phenomenon which is not yet fully understood in all the aspects. The first fundamental type of flutter is the coupled-mode flutter, which can occur for elastic structures with two degrees of freedom. If the natural frequencies of the respective modes (without airflow) are not far apart, when the object is subjected to airflow and

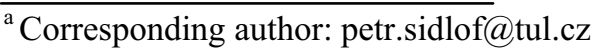

the flow velocity increases, the frequencies of the modes approach. For a critical flow velocity the frequencies merge and coupled-mode flutter instability occurs. The second common type of aeroelastic instability is the stall flutter. Here, the physical mechanism is different. The stall flutter of airfoils is triggered for high angles of attack, when the airflow separates from the airfoil surface. In this case, the ratio of the vortex shedding frequency and natural frequency of the airfoil vibration become important.

The current study reports on a 2D CFD model of flow-induced vibration of a rigid airfoil with two degrees of freedom - pitch (rotation about the elastic axis) and plunge (vertical deflection), supported by a linear and torsional spring. Such system can model a real aircraft wing, with pitching corresponding to torsional vibration of the elastic aircraft wing structure, and plunge corresponding to vibrations in the bending mode. The CFD model was designed according to parameters of the physical model used for wind tunnel measurements [2-5], so that the results of the CFD simulations can be compared with the experimental results.

\section{Methods}

The computational model tries to fit as well as possible the geometry, mechanical and aerodynamic parameters of the physical airfoil model depicted in Figure 1. The physical setup in Figure 1 is a redesigned and improved version of a model reported in [2]. The airfoil, supported by a linear and torsional springs, is fixed in a suction-type wind tunnel with optical access through the side walls for 
Schlieren visualizations and interferometric measurements of the flow field.

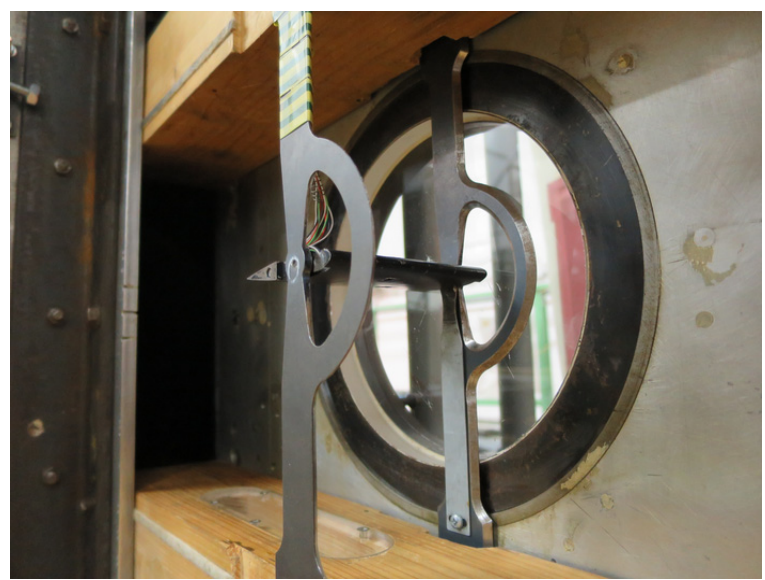

Figure 1. Physical model of the pitch-plunge airfoil in the wind tunnel (with front wall dismantled).

\subsection{Geometry and mesh}

The airfoil has a symmetric NACA0015 profile with a chord length of $59.5 \mathrm{~mm}$. The airfoil is located in a $2 \mathrm{D}$ channel with a height of $210 \mathrm{~mm}$ and length of $500 \mathrm{~mm}$ (measured from the trailing edge).

The computational grid for the CFD simulations is a C-type structured mesh generated in OpenFOAM's structured mesh generator blockMesh using an octave script provided in [6]. The mesh is refined towards the airfoil surface. For the purpose of first testing of the fluid-structure interaction simulations, a coarse mesh composed of only $10 \mathrm{k}$ quadrilateral elements was used in order to keep the simulation time as low as possible. The mesh is depicted in Figure 2.

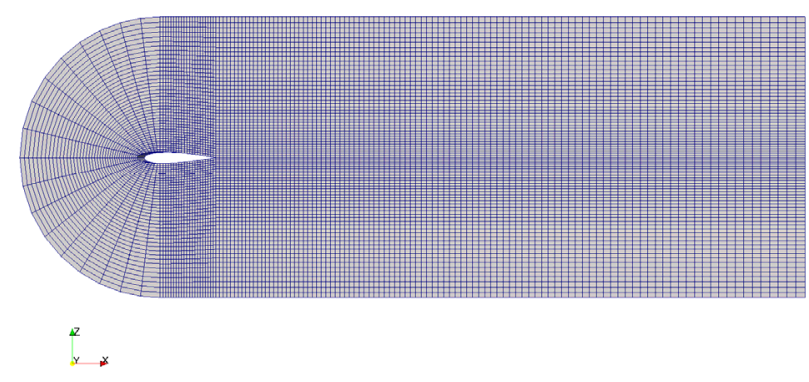

Figure 2. Geometry of the computational domain and mesh used for CFD simulations.

\subsection{Mechanical parameters}

All the mechanical parameters of the computational model were set according to the values directly measured or identified from the physical airfoil model. The elastic axis of the model is located in $1 / 3$ of the chord length slightly upstream of the centroid, located at $x_{T}=26.4 \mathrm{~mm}$. The mass of the airfoil and the moving parts of the frame (leading rods) is $m_{t}=1.498 \mathrm{~kg}$, the mass of the airfoil itself $m=148 \mathrm{~g}$. The natural frequencies of the plunging and pitching mode are $f_{1}=16.8 \mathrm{~Hz}$ and $f_{2}=14.5 \mathrm{~Hz}$, respectively. The linear stiffness of the support was measured as $k_{t}=16500 \mathrm{~N} / \mathrm{m}$. The computational model does not consider the total mass $m_{t}$, but the mass of the airfoil only. Thus, a reduced stiffness $k=1629 \mathrm{~N} / \mathrm{m}$ preserving the same natural frequency $f_{l}$ was used in the computational model. The moment of inertia of the airfoil was identified from the torsional natural frequency and torsional stiffness as

$$
J=\frac{k_{T}}{\left(2 \pi f_{T}\right)^{2}}=4.78710^{-5} \mathrm{~kg} \mathrm{~m}^{2} .
$$

A problematic issue is the damping of the respective modes. During parameter identification, the damping ratios were identified as $b_{l i d}=5.606 \mathrm{~N} \mathrm{~s} / \mathrm{m}$ and $b_{2 i d}=0.000785 \mathrm{~N} \mathrm{~m} \mathrm{~s} / \mathrm{rad}$. However, the mechanical design of physical model does not ensure that the damping of both modes is precisely linear, which resulted in discrepancies in vibration attenuation when using these values of the damping ratios in the computational model. Eventually, the ratios were set to $b_{l}=1.5 \mathrm{~N} \mathrm{~s} / \mathrm{m}$ and $b_{2}=0.0001 \mathrm{~N} \mathrm{~m} \mathrm{~s} / \mathrm{rad}$ to match the attenuation of the physical model for zero flow velocity.

\subsection{CFD model for the fluid-structure interaction, initial and boundary conditions}

The fluid flow is modeled by incompressible NavierStokes equations on the 2D computational domain

$$
\begin{aligned}
& \nabla \cdot \mathbf{u}=0 \\
& \frac{\partial}{\partial t} \mathbf{u}+\nabla \cdot(\mathbf{u} \otimes \mathbf{u})-v \Delta \mathbf{u}+\frac{1}{\rho} \nabla p=0
\end{aligned}
$$

for the flow velocity $\boldsymbol{u}$ and pressure $p$, with the kinematic viscosity of air $v=1.5810^{-5} \mathrm{~m}^{2} / \mathrm{s}$. No turbulence modeling was used in the CFD simulations published in this paper.

The boundary conditions imposed on the pressure and velocity fields were chosen to approximate the wind tunnel conditions. The air flow is driven by a constant velocity at inlet, which was measured by a Pitot tube in the physical setup. Due to the presence of large vortical structures convected downstream up to the outlet of the computational domain and consequent possible backflow to the domain, which destabilizes the numerical solution, a stabilized outflow condition was specified at the outlet of the domain: $\delta \mathbf{u} / \delta \mathbf{n}=0$ if the velocity direction points outward from the domain, and $\mathbf{u}=0$ otherwise. On the top and bottom walls a no-slip boundary condition was specified, on the airfoil surface, the flow velocity was set equal to the velocity of the structure. The pressure $p=0$ at outlet, on the other parts of the boundary $\delta p / \delta \mathbf{n}=0$.

The initial conditions were zero pressure and constant velocity in the whole computational domain. The initial position of the airfoil was set to $z=3 \mathrm{~mm}$, simulating the initial vertical deflection imposed to the physical model during wind-tunnel measurements. Additionally, the 
airfoil was given an initial angular velocity $\omega=-15.76 \mathrm{rad} / \mathrm{s}$ (corresponding to harmonic rotation with an amplitude of 10 degrees) to trigger the rotational mode.

The computational domain is deformed in time due to airfoil motion. The position of the airfoil and the coordinates of the element vertices in a new timestep are resolved using the following algorithm:

- With the knowledge of the pressure and velocity fields, the aerodynamic drag and lift force and the aerodynamic moment on the airfoil surface (both viscous and pressure parts) are calculated.

- The new position of the airfoil is found by solving the 2-DOF equation of motion of the system, including the external aerodynamic forces.

- The new position of the mesh vertices is calculated by a specialized OpenFOAM mesh motion solver based on spherical linear interpolation, which is designed to prevent cell shearing.

\subsection{Numerical solution}

The numerical solution was implemented in OpenFOAM, an object-oriented open-source set of $\mathrm{C}++$ libraries for CFD simulations. OpenFOAM is based on the finitevolume method in a collocated cell-centered approach on unstructured meshes. The incompressible Navier-Stokes equations on a moving computational mesh were solved numerically using a modified PISO algorithm [7]. In contrast to the standard PISO algorithm, it has a substep iteration loop: multiple cycles over the same timestep with the last iteration results (optionally under-relaxed) used as an initial guess for the next substep iteration.

The discretization schemes were as follows: implicit first-order Euler for the time derivative, linear upwind scheme for the convective term, and central differencing scheme (CDS) with nonorthogonal correction for the diffusion term. The timestep $\Delta t$ is adjusted automatically during the transient solution so that the maximum local Courant number is kept below a predefined limit. The Courant number on unstructured 3D meshes is calculated as

$$
C o=\frac{1}{2} \frac{\sum_{f}\left|\Phi_{f}\right|}{\Delta V} \Delta t,
$$

where $\Phi_{f}=A_{f}\left(\mathbf{u}_{f} \cdot \mathbf{n}_{f}\right)$ is the velocity flux normal to face $f$ with the surface $A_{f}$ of a cell with a volume $\Delta V$.

Due to significant element distortion and consequent loss of element orthogonality in the proximity of the moving airfoil, two outer loops of nonorthogonal correctors were utilized within the modified PISO algorithm to guarantee stability of the computation. Unlike the discretization of the temporal, convective and diffusive terms, the nonorthogonal correctors are treated explicitly. The numerical scheme thus acquires explicit character and the timestep has to be limited so that the CFL condition is satisfied. In the current simulations, the Courant number was limited to $\mathrm{Co}<0.9$.
The resulting linear system for momentum was solved using a Gauss-Seidel smoother. For the pressure predictor and corrector steps and for the mesh motion, a geometric multigrid solver was used with an OpenFOAM-specific cell agglomeration algorithm and a conjugate-gradienttype method for solution of the coarsest level matrix.

The simulations were run in parallel using the domain decomposition method. The current simulations on a coarse mesh consisting of 10k elements proved efficient parallel speedup up to four CPU cores on a SGI Altix UV-100 shared-memory supercomputer.

\section{Results}

To reveal the stability boundaries of the system and determine the critical flow velocity for the onset of instable exponentially increasing airfoil vibration, the numerical simulations were run for inflow velocities $u=30-90 \mathrm{~m} / \mathrm{s}$ (see Table 1$)$.

Table 1. Inlet flow velocity $u[\mathrm{~m} / \mathrm{s}]$ in numerical simulations s520a .. s520g. The airfoil oscillations are stable for s520a-e, unstable for s520f-g.

\begin{tabular}{|l|c|c|c|c|c|c|c|}
\hline $\mathbf{s 5 2 0}$ & $\mathrm{a}$ & $\mathrm{b}$ & $\mathrm{c}$ & $\mathrm{d}$ & $\mathrm{e}$ & $\mathrm{f}$ & $\mathrm{g}$ \\
\hline $\boldsymbol{u}[\mathbf{m} / \mathbf{s}]$ & 30 & 40 & 50 & 60 & 70 & 80 & 90 \\
\hline
\end{tabular}

For the velocities $u=30-70 \mathrm{~m} / \mathrm{s}$, the oscillations remain stable and exponentially decay due to the mechanical damping in the system (see Figure 3). For inflow velocities exceeding $80 \mathrm{~m} / \mathrm{s}$, however, the system is instable and the amplitudes exponentially increase in time. The CFD simulations are limited by the extent of airfoil motion, which induces mesh deformation. Under very large amplitudes, especially in the angle of attack, the computational mesh deforms too much, the elements distort and the numerical simulation becomes unstable. This happened in s520f and s520g for pitching angles exceeding about 50 degrees.
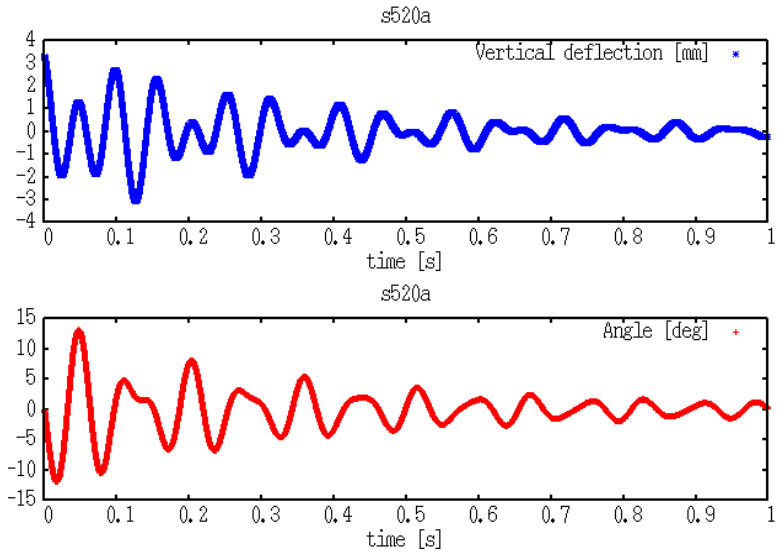

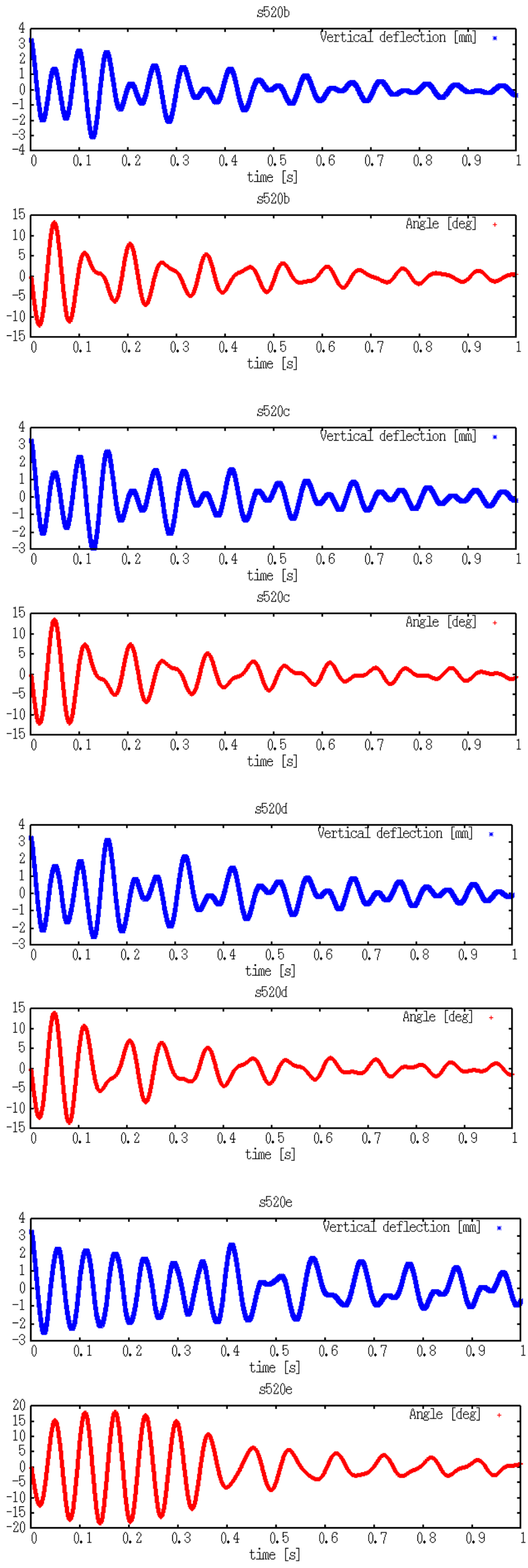
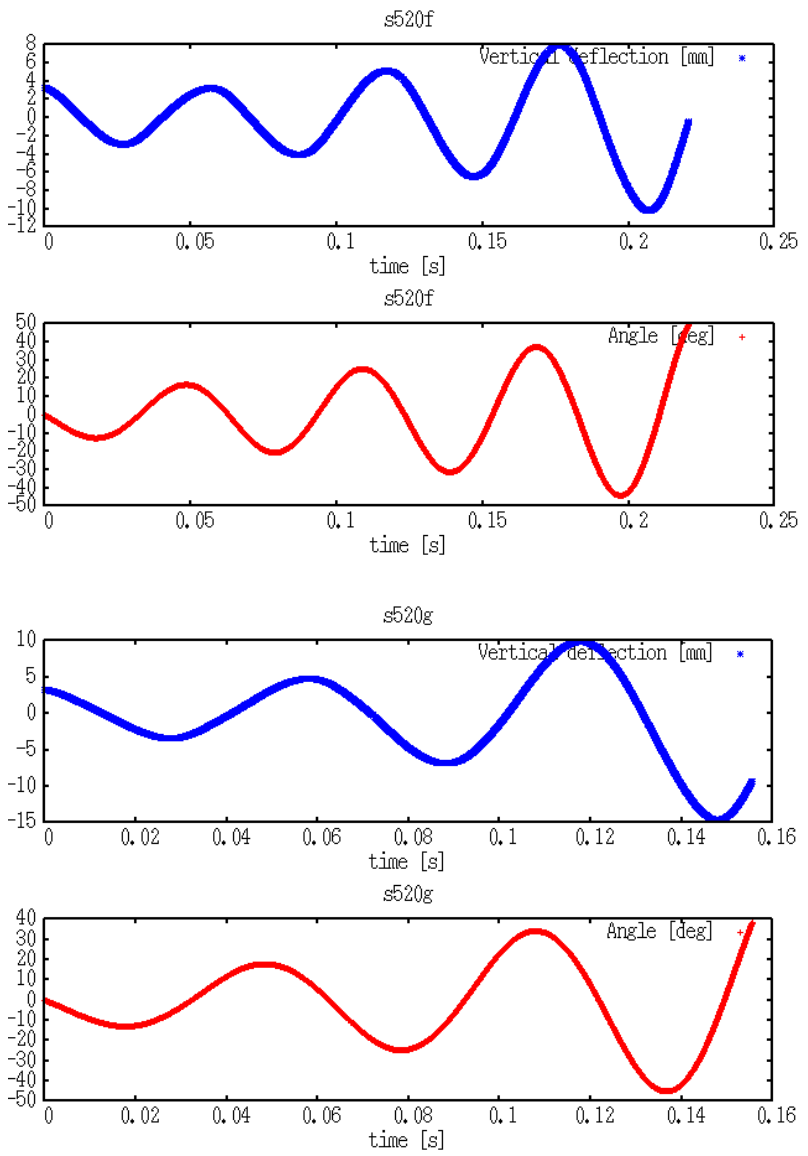

Figure 3. Time history of plunge and pitch in numerical simulations s520. Stable damped oscillations (s520a..s520e), instable exponentially increasing amplitudes (stall flutter, s520f and $\mathrm{s} 520 \mathrm{~g}$ )
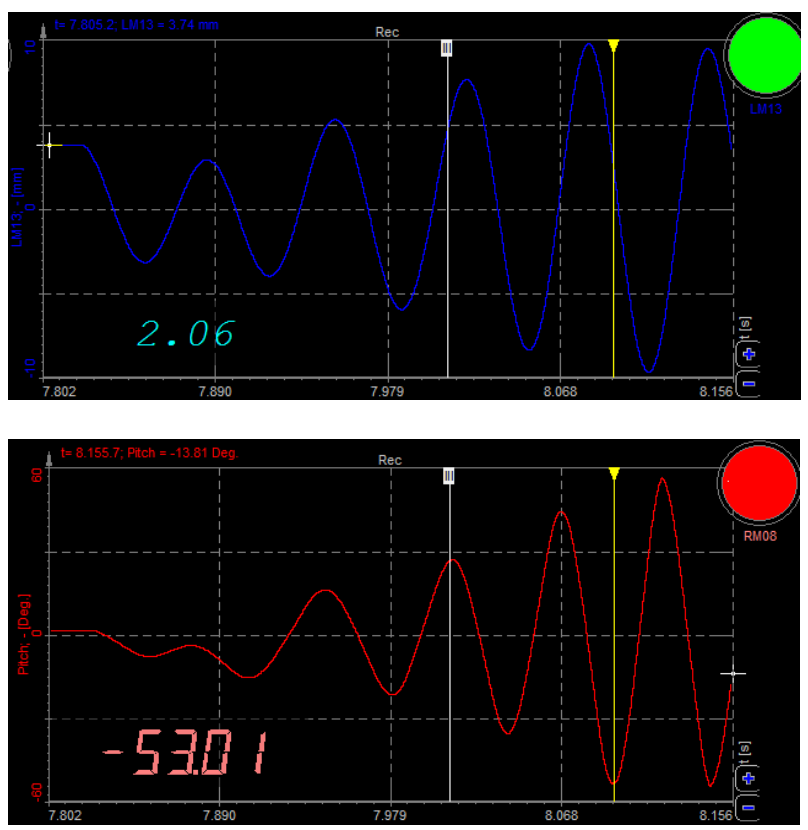

Figure 4. Time history of plunge and pitch in wind-tunnel measurement No. 2915-22 (inlet Mach number $\mathrm{Ma}=0.228$, $\mathrm{u}=78.2 \mathrm{~m} / \mathrm{s}$. After about two seconds of the transient development with amplitudes reaching $10 \mathrm{~mm}$ and $56 \mathrm{deg}$, the amplitudes stabilize at $5.9 \mathrm{~mm}$ and $42 \mathrm{deg}$ (not shown in figure). 
The simulated time history of pitch and plunge in Figure 3 can be compared with values measured on the physical model in the wind tunnel. Figure 4 shows the time development of the vertical deflection and angle of attack in measurement 2915-22, where the inlet Mach number was $\mathrm{Ma}=0.228$ (inflow velocity $u=78.2 \mathrm{~m} / \mathrm{s}$ ), corresponding approximately to numerical simulation s520f. The numerical simulation is in quite good agreement with the measurement, only the amplitudes in the measurement are slightly higher.

The velocity fields from numerical simulation s520f are shown in Figure 5 in time instants corresponding to zero, minimum and maximum pitching angles. The simulation shows the vortex street generated in the wake of the airflow. For the higher angles of attack, the flow is massively separated with vortex structures shed from the shear layer.
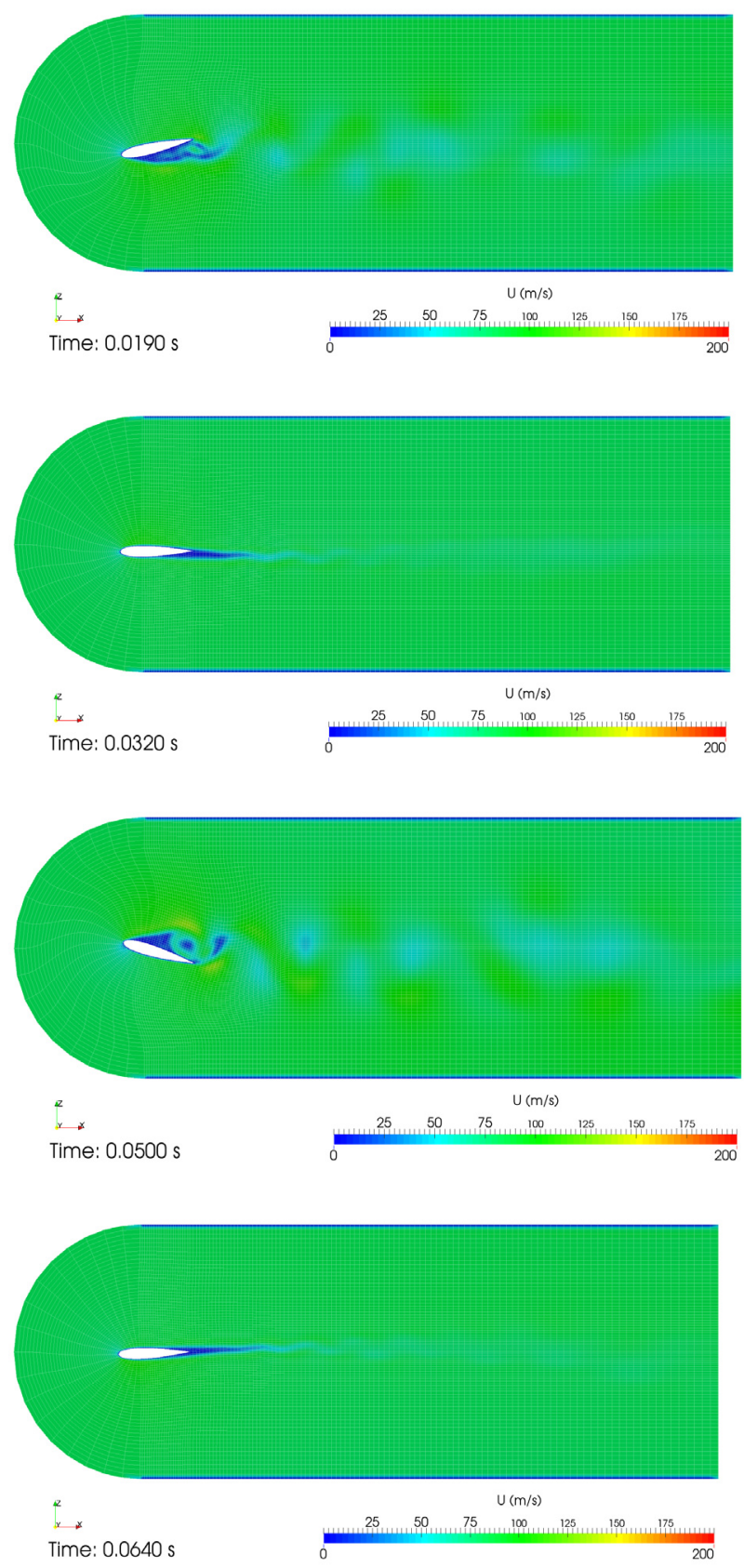
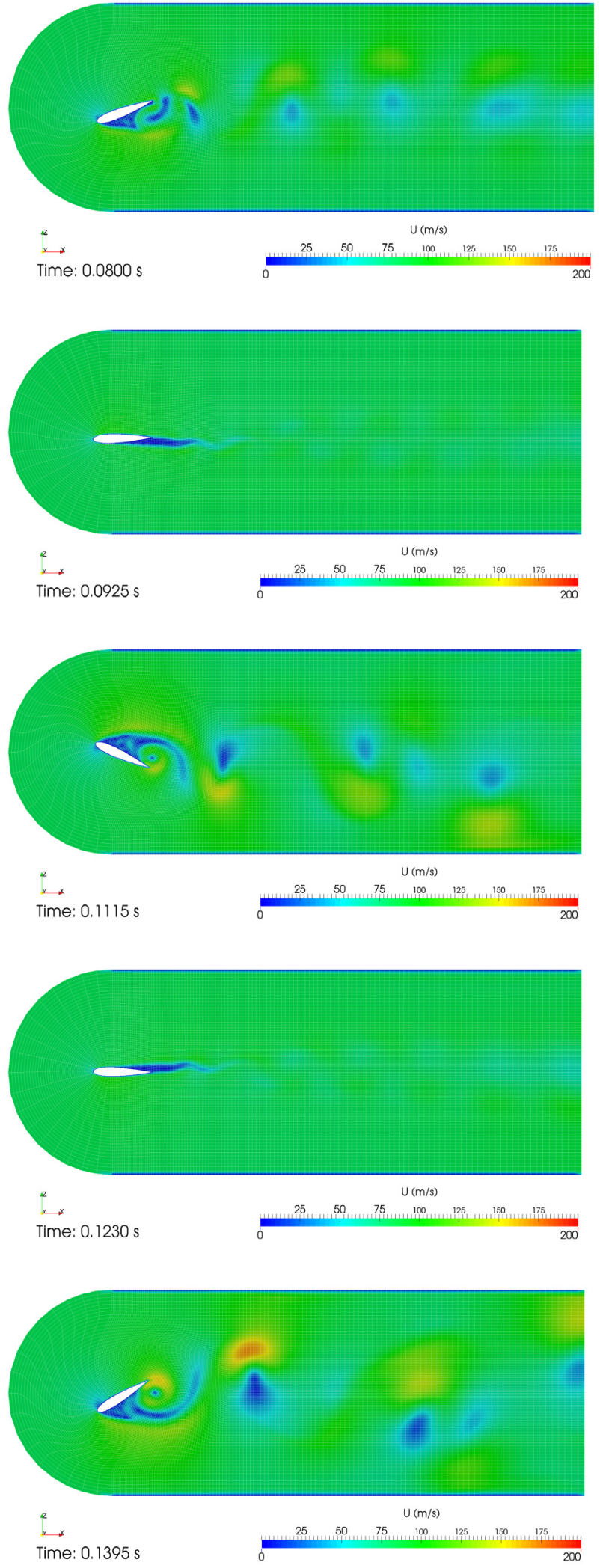

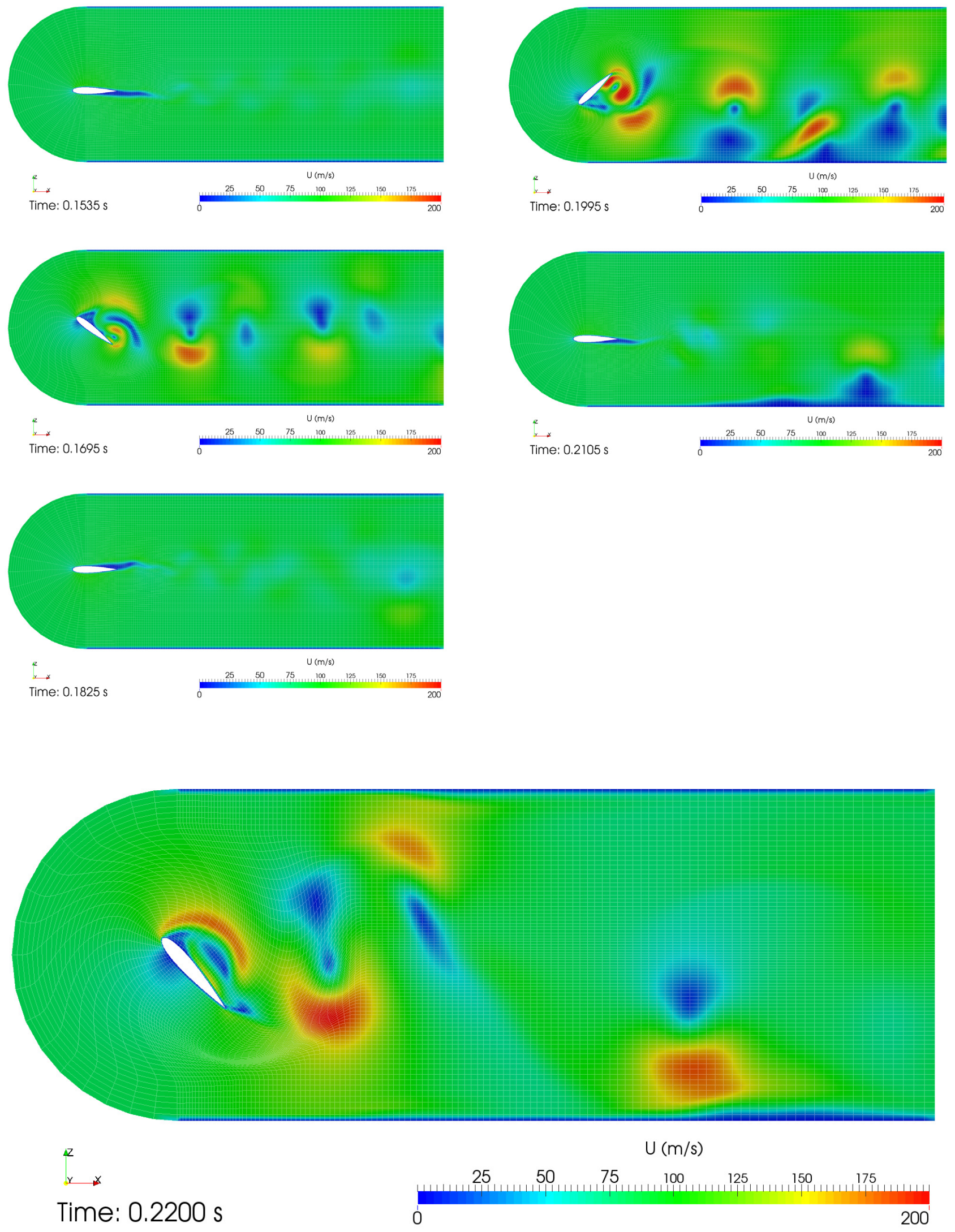

Figure 5. Time development of the velocity magnitude field in simulation s520f ( $u=80 \mathrm{~m} / \mathrm{s}$ ). 


\section{Discussion}

Taking into account that the numerical simulation is performed on a very coarse 2D mesh and that it contains numerous simplifications, the results compare to the experimental data unexpectedly well. Without any artificial parameter tuning, the critical flow velocity predicted by the numerical simulation lies between $70-$ $80 \mathrm{~m} / \mathrm{s}$, while the measurements revealed the flutter boundary at about $65 \mathrm{~m} / \mathrm{s}$. The time histories of the pitching and plunging motion also match very well.

\subsection{Limitations of the current approach}

The incompressible flow assumption holds reasonably for flow velocities up to $\mathrm{Ma}=0.3$, and thus the incompressible flow model used in the simulations is on the verge on its validity. In the current approach, no turbulence modeling was considered. Further, the computational grid is too coarse to capture accurately the boundary layer on the airfoil. Due to drastic differences between the computational requirements of $2 \mathrm{D}$ and $3 \mathrm{D}$ simulations, the first tests were run in 2D only. Especially in the downstream section of the geometry, the predicted 2D flow fields necessarily depart from the 3D reality due to fundamentally different vortex dynamics in two and three dimensions.

\subsection{Future steps}

The computational model is ready for parallel numerical simulations in $3 \mathrm{D}$. In previous parallel speedup testing [8], the code proved good scaling up to $40 \mathrm{CPU}$ cores for meshes consisting of about three million elements. The $\mathrm{k}-\omega$ SST turbulence model and the compressible flow model have already been successfully used in previous work [9], and can be included in further simulations.

\section{Conclusions}

Both experiments and simulations show that the instability onset is sensitive to the initial conditions. The instability is clearly of the stall-flutter type, which is driven by the flow separation on the suction surface of the airfoil. To trigger the pitching motion and thus flow separation, the numerical simulations needed an initial impulse in angular velocity, otherwise the pitching angles remained too small and the instability did not occur even for higher flow velocities.

\section{Acknowledgements}

The research has been supported by the Czech Science Foundation, project 13-10527S "Subsonic flutter analysis of elastically supported airfoils using interferometry and CFD".

The parallel scaling tests were run on the facilities of the Supercomputing center at the Czech Technical University in Prague. The experimental data were acquired during measurements in the wind tunnel of the
Institute of Thermomechanics, Academy of Sciences of the Czech Republic in Nový Knín, in cooperation with Martin Štěpán, Václav Vlček, Martin Luxa and David Šimurda. The natural frequencies and damping ratios of the mechanical model were measured by Štěpán Chládek.

\section{References}

1. E.H. Dowell, A Modern Course in Aeroelasticity (Kluwer Academic Publishers, Dodrecht, 1995)

2. P. Šidlof, V. Vlček, M. Štěpán, J. Horáček, M. Luxa, D. Šimurda, J. Kozánek, Proceedings of the ASME PVP 2014 conference, ASME, 2014

3. P. Šidlof, M. Štěpán, V. Vlček, V. Řidký, D. Šimurda, J. Horáček, EPJ Web of Conferences 67, 02108 (2014)

4. M. Štěpán, Proceedings of Colloquium DYNAMESI 2014, Institute of Thermomechanics, (2014)

5. V. Vlček, M. Štěpán, I. Zolotarev, J. Kozánek, Proceedings of Engineering Mechanics 2015, pp. 350-351 (2015)

6. https://www.hpc.ntnu.no/display/hpc/OpenFOAM++Airfoil+Calculations, accessed 2015-30-07

7. J.H. Ferziger, M. Peric, Computational Methods for Fluid Dynamics (Springer, Berlin, 2002)

8. P. Šidlof, V. Řidký, EPJ Web of Conferences 92, 02080 (2015)

9. ̌ridký V., Šidlof P., Engineering Mechanics 2014, pp. 548-551, (2014) 